\title{
Avances y aplicaciones en física de rocas para exploración de hidrocarburos
}

\section{Advances and Applications of Rock Physics for Hydrocarbon Exploration}

\author{
Vargas-Meleza L. \\ Gerencia de Prospección Geofísica \\ Dirección de Exploración y Producción \\ Instituto Mexicano del Petróleo (IMP) \\ Correo:lvargasm@imp.mx
}

\author{
Valle-Molina C. \\ Gerencia de Prospección Geofísica \\ Dirección de Exploración y Producción \\ Instituto Mexicano del Petróleo (IMP) \\ Correo:cvallem@imp.mx
}

Información del artículo: recibido: enero de 2011, aceptado: enero de 2012

\section{Resumen}

Se discuten los fundamentos de física de rocas y las implicaciones analíticas para interpretación sísmica de yacimientos. Se considera conveniente difundir, entre los ingenieros y estudiantes mexicanos, los fundamentos y metodologías actuales sobre el análisis de la física de rocas en exploración de hidrocarburos. Este trabajo representa un esfuerzo de capacitación profesional en exploración petrolera en el que se difunde la relevancia de la física de rocas. El interés principal es exponer los avances tecnológicos y aplicaciones actuales sobre física de rocas en el campo de sismología de exploración. La mayoría de las metodologías estudia los mecanismos físicos y geológicos que controlan las propiedades elásticas de los yacimientos de hidrocarburos, a partir de núcleos de roca y registros geofísicos de pozo. Este conocimiento se usa para predecir propiedades de la roca (litología, porosidad, tipo de fluido) mediante datos sísmicos tridimensionales que se sugiere deben ser debidamente calibrados con mediciones experimentales de laboratorio e información de pozos. La aplicación de metodologías integradas, las cuales incorporan información geológica y geofísica, ayuda a establecer las relaciones entre propiedades petrofísicas y elásticas del yacimiento, incorporando informaciones de diferente naturaleza y escala.

\section{Descriptores:}

- física de rocas

- caracterización dinámica de núcleos de roca

- sismología de exploración

- ingeniería geofísica

- exploración petrolera 


\begin{abstract}
Integration of the geological and geophysical information with different scale and features is the key point to establish relationships between petrophysical and elastic characteristics of the rocks in the reservoir. It is very important to present the fundamentals and current methodologies of the rock physics analyses applied to hydrocarbons exploration among engineers and Mexican students. This work represents an effort to capacitate personnel of oil exploration through the revision of the subjects of rock physics. The main aim is to show updated improvements and applications of rock physics into seismology for exploration. Most of the methodologies presented in this document are related to the study the physical and geological mechanisms that impact on the elastic properties of the rock reservoirs based on rock specimens characterization and geophysical borehole information. Predictions of the rock properties (litology, porosity, fluid in the voids) can be performed using $3 D$ seismic data that shall be properly calibrated with experimental measurements in rock cores and seismic well log data.
\end{abstract}

\section{Introducción}

Debido a la complejidad de los yacimientos de hidrocarburos actuales, es necesario mejorar el conocimiento sobre los mecanismos físicos y geológicos que rigen las propiedades mecánicas y constantes elásticas de las rocas, por lo que el conocimiento y aplicación de física de rocas es importante. Este esfuerzo de difusión está dirigido a los ingenieros de la industria petrolera mexicana y a los estudiantes de posgrado en centros de educación superior en México. Uno de los intereses de este trabajo radica en presentar los avances tecnológicos y aplicaciones actuales de física de rocas para predicción de propiedades de los yacimientos (por ejemplo, litología, tipo de fluido, saturación) a partir de amplitudes y velocidades sísmicas.

Las propiedades físicas de rocas y minerales se han estudiado desde hace décadas, en el contexto de exploración sísmica del subsuelo; desde entonces, se han realizado experimentos y han surgido teorías que buscan correlacionar las amplitudes y velocidades sísmicas con la litología del subsuelo. Actualmente, los datos sísmicos se analizan para determinar litología, porosidad, tipo de fluido y saturación, con base en las relaciones entre las velocidades de propagación de ondas sísmicas y propiedades elásticas de la roca. En el presente trabajo, se describen los factores más importantes que afectan a las velocidades de propagación y sus implicaciones en la interpretación de propiedades del yacimiento.

La interpretación sísmica cuantitativa se está convirtiendo en una de las herramientas principales de la física de rocas. Los modelos derivados de este análisis físico, a partir de información in situ de los registros de pozos, se utilizan para extrapolar las propiedades de la roca hacia zonas alejadas de los pozos existentes, con ello se facilita la exploración de nuevas áreas de oportunidad. Existen diversos modelos de física de rocas, cada uno con sus ventajas y limitaciones, pero discutir cada uno de estos modelos está fuera del objetivo de este trabajo.

Algunas de las aplicaciones más importantes del análisis de física de rocas son: inversión de datos sísmicos para convertir series de reflectividad en propiedades de la rocas, como impedancia acústica, porosidad, volumen de arcilla, etcétera (Kemper, 2010; Xin-Gang y De-Hua, 2009); cuantificación de propiedades de las rocas para caracterizar el yacimiento mediante análisis estadísticos multivariables (Avseth et al., 2007; Doyen, 2009); caracterización dinámica de especímenes de roca empleando técnicas experimentales (King, 2009) y recientemente, modelado digital, tridimensional, que permite simular diferentes tipos de flujo y observar el efecto simultáneo de varios factores sobre los valores de velocidad (Knackstedt et al., 2009; Dvorkin y Nur, 2009).

Adicionalmente, la aplicación de conceptos de física de rocas en sismología de exploración involucra dos problemas principales:

- establecer relaciones entre los fenómenos físicos y el comportamiento elástico de las rocas,

- integrar datos de naturaleza y resolución vertical diferentes para predecir propiedades de la roca.

En el primer caso, propiedades como litología, porosidad, permeabilidad, tipo de fluido, grado de saturación y tipo de poros se pueden medir directamente en muestras de roca realizadas en laboratorio o in situ, mediante registros geofísicos de pozos. Las relaciones o tendencias entre las propiedades de la roca y las geofísicas, tienen dos orígenes principales: el régimen de 
compactación y el ambiente geológico de depósito (Avseth et al., 2010). En el segundo caso es necesario encontrar un plano equivalente para analizar datos observados a diferente frecuencia o escala.

En la figura 1 se muestran las diferentes técnicas sísmicas y su correspondiente resolución vertical. Por una parte, el análisis de núcleos de roca y los registros geofísicos de pozo proveen información de alta resolución, por ejemplo, en milímetros a decenas de centímetros, por lo que estudian un volumen reducido de roca. Por otra parte, los métodos sísmicos de superficie observan un volumen mayor de roca como decenas a cientos de metros, pero con resolución limitada. Las mediciones sísmicas entre pozos pueden disminuir la brecha entre mediciones geofísicas de resolución alta y limitada (Harris y Langan, 1997).

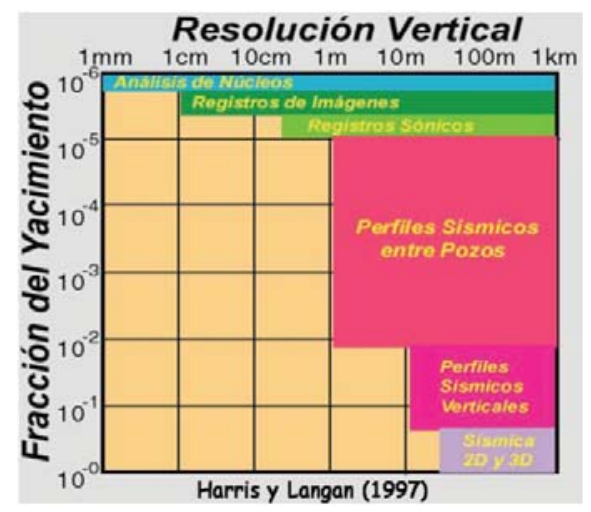

Figura 1. Variación de la resolución vertical asociada con las diferentes técnicas de exploración geofísica, con respecto a la fracción detectable de yacimientos de hidrocarburos (modificado de Harris y Langan, 1997).

Adicionalmente, este trabajo forma parte de un esfuerzo de capacitación profesional que se origina a partir de nuestra interacción continua con los profesionales de PEMEX, Exploración y Producción, a través de su Gerencia de Geofísica, quienes mostraron interés en obtener capacitación sobre temas de física de rocas para atacar las necesidades actuales de la industria petrolera mexicana. Por tanto, preparamos cursos de capacitación impartidos por medio de talleres tecnológicos sobre aspectos fundamentales (teóricos y prácticos) de caracterización de núcleos y física de rocas en exploración. En estos cursos tratamos técnicas experimentales de vanguardia para caracterización dinámica de muestras de roca y metodologías integradas de actualidad, utilizadas tanto por instituciones académicas como por empresas petroleras de ámbito mundial para estimar propiedades del yacimiento a partir de datos sísmicos. Nuestra experiencia de colaboración con PEMEX, Ex- ploración y Producción, nos permitió identificar las brechas de conocimiento y tecnológicas en materia de física de rocas. Con ello pudimos elaborar el material de este trabajo.

Los temas que se abordan a lo largo de este documento son conceptos de elasticidad y su relación con velocidades de propagación de ondas. Entonces, describimos dos técnicas experimentales con las que se caracterizan dinámicamente los núcleos de roca. Discutimos también aspectos sobre modelado de velocidades de propagación y algunos de los principales factores físicos que afectan su comportamiento. Finalmente, presentamos una metodología generalizada de aplicación de modelos de física de rocas y sísmica, así como avances tecnológicos en la caracterización de yacimientos y su impacto en la exploración de hidrocarburos.

\section{Antecedentes}

La aplicación de sismología para describir las rocas y sus fluidos tiene fundamento en la propagación de ondas elásticas, fenómeno descrito por la ecuación 1.

$\rho \frac{\partial^{2} u}{\partial t^{2}}=\left(\lambda+\frac{4}{3} \mu\right) \nabla(\nabla \cdot u)-\mu \nabla \times(\nabla \times u)$,

$V_{P}=\sqrt{\frac{\lambda+\frac{4}{3} \mu}{\rho}}$,

$V_{S}=\sqrt{\frac{\mu}{\rho}}$,

donde

$u$ es el campo de desplazamiento,

$t$ es tiempo,

$\rho$ es densidad y

$\lambda$ y $\mu$ los parámetros de Lamé de incompresibilidad y rigidez, respectivamente.

Esta ecuación relaciona las velocidades de propagación de ondas de compresión $\left(V_{P}\right)$ y de corte $\left(V_{S}\right)$ con los parámetros de Lamé, como se indica en las ecuaciones 2 y 3. Así, las velocidades de propagación asociadas a los diferentes módulos elásticos son la clave para definir información del subsuelo a partir de datos sísmicos. Por su parte, las señales sísmicas contienen información del contraste de propiedades elásticas del subsuelo en forma de tiempos de arribo, variaciones de amplitud y fase.

El objetivo final es interpretar las amplitudes sísmicas en términos de litología, porosidad y tipo de fluido 
usando las funciones de transformación obtenidas en los modelos de física de rocas.

La rigidez de la roca se puede determinar mediante la medición de los módulos elásticos de compresión confinada $(M)$, el módulo de Young $(E)$ y el de rigidez al corte $(\mu)$. Las ecuaciones 4 a 6 muestran las relaciones entre las velocidades de propagación de ondas compresionales y de corte con los módulos elásticos, donde el módulo de Young se mide para condiciones no confinadas, dando lugar a una la velocidad de onda compresional, no confinada $\left(V_{C}\right)$.

$M=\rho V_{p}^{2}$,

$E=\rho V_{c}^{2}$,

$\mu=\rho V_{s}^{2}$.

Por otro lado, las velocidades de propagación $V_{P}$ y $V_{S}$ se pueden relacionar con la frecuencia de propagación de la onda $(f)$ y la longitud de onda $(\lambda)$ correspondientes, por medio de las relaciones siguientes:

$$
\begin{aligned}
& V_{P}=f_{P} \lambda_{P}=\sqrt{\frac{M}{\rho}}, \\
& V_{S}=f_{S} \lambda_{S}=\sqrt{\frac{\mu}{\rho}} .
\end{aligned}
$$

Finalmente, es posible determinar la relación de Poisson $(v)$ a partir de las velocidades de propagación $V_{P} \mathrm{y}$ $V_{S^{\prime}}$ como se indica en la ecuación 9,

$$
\frac{\mathrm{V}_{\mathrm{p}}}{\mathrm{V}_{\mathrm{S}}}=\sqrt{\frac{2(1-v)}{(1-2 v)}} .
$$

\section{Técnicas experimentales para caracterización de rocas}

En esta sección describimos dos técnicas experimentales usadas en laboratorio para caracterizar núcleos de roca. Una de ellas se basa en mediciones de resonancia y la otra en mediciones sísmicas. La primer técnica mide las velocidades de propagación de ondas de cuerpo utilizando la curva de respuesta de los especímenes, que es la variación de la respuesta de la muestra de roca con la frecuencia (Stokoe et al., 1994a). Las pruebas de resonancia permiten evaluar la rigidez $(\mu)$ y el amortiguamiento (D) del material (e indirectamente, el coeficiente de atenuación $Q$ ). La segunda es la técnica de tiempo directo de arribo de onda (onda P y onda S), también conocida como de pulso-velocidad o mediciones ultrasónicas (King, 2009). La medición de las velocidades de propagación de onda de compresión y corte permite, independientemente de la técnica experimental, caracterizar muestras de roca dinámicamente, determinando los diferentes módulos elásticos. Sin embargo, se deben tomar en cuenta las potenciales discrepancias causadas por los diferentes rangos de frecuencia de excitación y nivel de deformación utilizados en cada caso (King, 2009).

\section{Técnica de resonancia}

La técnica de resonancia consiste en excitar torsionalmente y longitudinalmente a los núcleos de roca por medio de impactos (Stokoe et al., 1994a y 1994b). Así, los especímenes entran en vibración (primer modo de vibración) y los acelerómetros, que se usan para monitorear el movimiento con respecto a la frecuencia, dan como resultado las curvas de respuesta de las muestras de roca. Típicamente, la prueba de resonancia puede realizarse en condiciones de frontera fijo-libre o libre-libre.

La configuración de la columna resonante (resonant column and torsional shear RCTS) para una prueba de resonancia de tipo libre-libre se ilustra en la figura 2. La frecuencia de resonancia y la amplitud de vibración se determinan a partir de la curva de respuesta. Estos valores se combinan con las características del equipo y el tamaño del espécimen para calcular velocidad de corte $\left(V_{S}\right)$, módulo de rigidez $(\mu)$ y amplitud de la deformación de corte, $(\gamma)$.

La prueba dinámica de resonancia realizada de manera libre-libre incluye mediciones: 1 ) resonancia librelibre y 2) tiempo directo de arribo. En la porción experimental de resonancia se aplica una excitación torsional para medir la velocidad de propagación de ondas de corte, $V_{S}$, el módulo de rigidez, $\mu_{\max }$, y el amortiguamiento correspondiente, $D_{\text {Smin }}$. Similarmente, con una excitación longitudinal se miden la velocidad de propagación de ondas compresionales, $V_{C}$, el módulo de Young, $E_{\max }$, y el amortiguamiento en condiciones no confinadas. $D_{C m i n}$.

Básicamente, se determinan las frecuencias de resonancia del primer modo de vibrar a partir de la curva de respuesta como la que se observa en la figura 3. Para el caso libre-libre la relación entre las velocidades y las frecuencias de resonancia se determinan con las ecuaciones 10 y 11a través de la longitud de onda.

$V_{C}=f_{1} \lambda_{C}$

$V_{S}=f_{1} \lambda_{S}$ 
donde

$f_{1}=$ frecuencia de resonancia del primer modo (por una excitación longitudinal para $V_{C} \mathrm{y}$ torsional para $V_{S}$ ) $\mathrm{y}$

$\lambda=$ longitud de onda para una barra libre-libre.

Las relaciones de amortiguamiento de los materiales para condiciones de compresión no confinadas $\left(D_{C \text { min }}\right)$ y

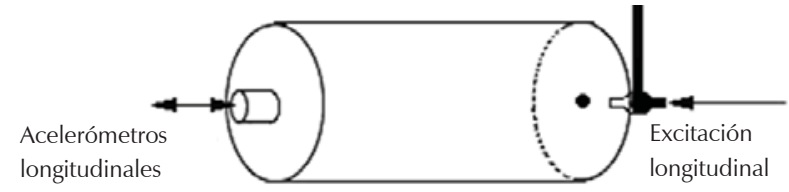

a) Prueba confinada de onda compresional

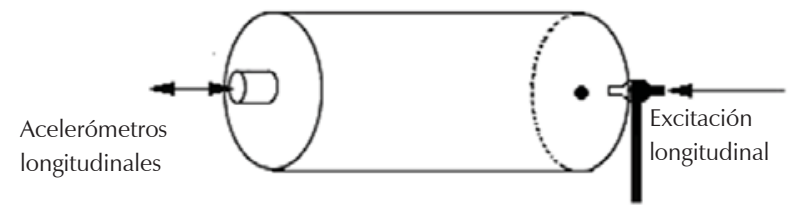

b) Prueba de resonancia compresional

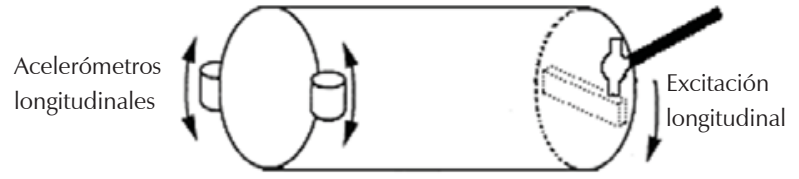

c) Prueba de resonancia torsional

Figura 2. Configuración para a) medición de tiempo directo de arribo, b) y c) prueba de resonancia libre-libre, en condiciones no confinadas y en especímenes cilíndricos de roca para determinar velocidades $\mathrm{V}_{\mathrm{p}}$ y $\mathrm{V}_{\mathrm{S}}$ (modificado de Stokoe et al., 1994b).

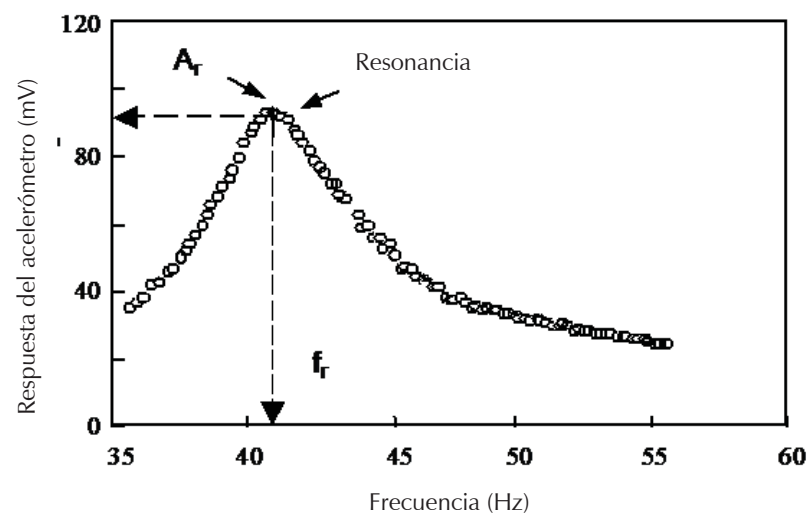

Figura 3. Curva de respuesta dinámica medida en una prueba de columna resonante, donde $A_{r}$ es la amplitud de resonancia y $f_{r}$ la frecuencia de resonancia (Stokoe et al.,1994a) torsional $\left(D_{S \text { min }}\right)$, se pueden determinar a partir de la amplitud de la curva de respuesta usando la técnica de ancho de banda (half power bandwith method).

Por otra parte, los tiempos directos de arribo de la onda compresional se miden entre los dos extremos del espécimen para estimar la velocidad de propagación, confinada, $V_{p}$, cuidando que la excitación longitudinal ocurra al centro del espécimen para asegurar el confinamiento. En la figura 2 se ilustran estos tres tipos de mediciones. En todos los casos, el espécimen está bajo condiciones no confinadas y todos los niveles de deformación se comportan linealmente.

La columna resonante de tipo fijo-libre tiene como principio de operación típico, vibrar el espécimen cilíndrico en el primer modo de resonancia torsional, utilizando un sistema de imanes y bobinas como se ilustra en la figura 4. La ventaja principal de este equipo, en comparación con la configuración libre-libre mostrada en la figura 2, radica en la posibilidad de aplicar confinamiento. El movimiento del espécimen se monitorea con un acelerómetro. Adicionalmente, este equipo permite realizar la prueba de torsión cíclica para bajas frecuencias en la que el movimiento se monitorea con proxímetros (Stokoe et al., 1994b).

Dicho equipo tiene dos características importantes: primero, tanto la columna resonante $(R C)$ como la prueba de torsión cíclica (TS) se pueden realizar con el mismo equipo. Para pasar de un modo de prueba al otro, simplemente se cambian, fuera de la cámara de confinamiento: 1) la frecuencia de excitación de inicio para excitar el espécimen, y 2) los transductores de monitoreo de movimiento que registran la respuesta del espécimen. Como resultado, la variabilidad debida a diferentes especímenes se elimina para hacer compara-

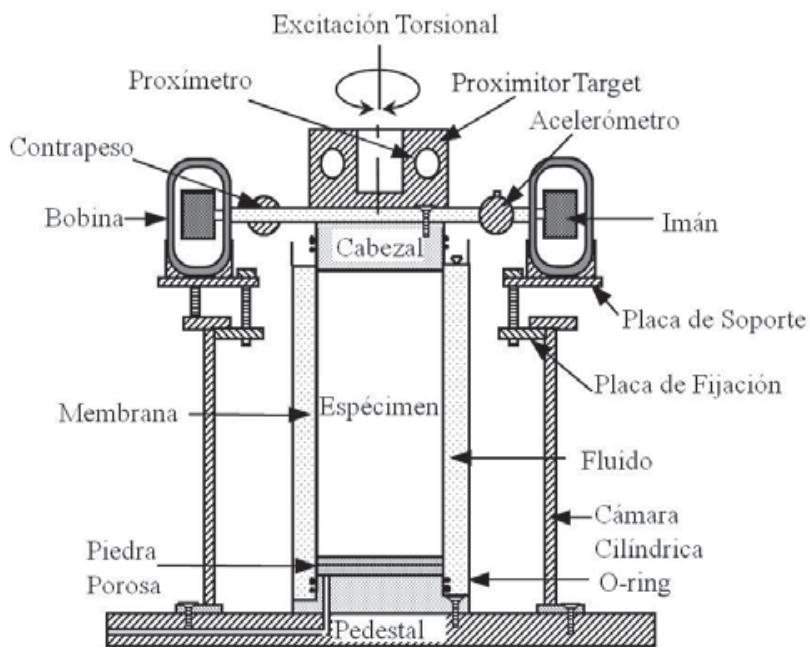

Figura 4. Equipo de columna resonante y torsión cíclica, RCTS (modificado de Stokoe, et al.,1994a) 
ble las pruebas. Segundo, la frecuencia de excitación en la prueba de torsión cíclica varía entre $0.1 \mathrm{~Hz}$ y $10 \mathrm{~Hz}$, por lo tanto, se puede investigar el efecto de frecuencia y el número de ciclos de carga observado en las propiedades dinámicas de especímenes de roca.

Finalmente, entre las ventajas del uso de la técnica de resonancia en laboratorio se encuentra el rango de frecuencia medido, que usualmente está entre 100 y 600 $\mathrm{Hz}$, lo cual cubre una brecha de resolución entre las mediciones ultrasónicas y sísmicas de exploración, así como la posibilidad de medir el amortiguamiento de la muestra de roca, y en consecuencia, se puede medir el factor de calidad $Q$, (o atenuación).

\section{Técnica de tiempo directo de arribo de onda}

Esta técnica para caracterización de núcleos de roca se realiza típicamente usando transductores piezoeléctricos. La piezoelectricidad es una propiedad que tienen ciertos materiales, que les permite transformar la energía mecánica en eléctrica y viceversa. Los materiales piezoeléctricos comúnmente utilizados como transductores son el cuarzo y el zirconato-titanato.

El rango de frecuencias en el que se miden las velocidades de propagación de las ondas $P$ y $S$ en mediciones ultrasónicas varía entre $100 \mathrm{KHz}$ y $1 \mathrm{MHz}$ (King, 2009). La aplicación de altas frecuencias de excitación obedece a la maximización del movimiento de las ondas que ocurre al coincidir la frecuencia aplicada con la frecuencia natural de vibración del transductor. La velocidad de propagación de las ondas depende de la frecuencia de excitación. En consecuencia, los valores de velocidad medidos en laboratorio no son únicos y dependen de las características del equipo y técnica experimental utilizados.

\section{Modelado de velocidades}

Las propiedades geofísicas de la roca (i.e. módulos elásticos y velocidades de propagación) dependen de la mineralogía, propiedades del fluido en los poros y de la geometría de la roca. Particularmente, para aplicaciones de sismología de exploración, nos interesa conocer la variación de velocidad asociada al cambio del esfuerzo efectivo, al tipo de fluido, al grado de saturación de agua, a la porosidad e, incluso, la frecuencia (Wang, 2001). Estos factores, entre otros, se encuentran interrelacionados de maneras complejas, de tal forma que la variación de uno de ellos produce cambios simultáneos en otras propiedades de la roca.

Los modelos de física de rocas son relaciones matemáticas que se usan para convertir las propiedades de la roca y el fluido que conforman el yacimiento en propiedades geofísicas. Modelar las propiedades geofísicas de las rocas responde a la necesidad de conocer su comportamiento en áreas en dónde no se cuenta con información de pozos. Así, un modelo se crea variando el tipo de fluido, presión efectiva, espesor del yacimiento, litología, porosidad, etcétera para investigar la sensibilidad de los datos sísmicos a estos factores. Con el modelado de velocidades se pueden resolver ambigüedades en los datos disponibles y por lo tanto, se puede también estimar la incertidumbre en los resultados.

Cualquier modelo de física de rocas debe ser calibrado y validado con experimentos controlados en laboratorio, mediciones de campo (registros geofísicos de pozos) para extender el rango de aplicación a datos sísmicos. Para ello, deben considerarse las características locales del yacimiento. El esfuerzo efectivo regula el proceso de compactación de los sedimentos, dando lugar a diferentes tipos y valores de porosidad (densidad) para una misma litología. Mientras, la velocidad de propagación de ondas elásticas depende de la estructura porosa, composición mineralógica, estado de esfuerzos y los fluidos que saturan la roca. En este sentido, es intuitivo relacionar velocidad de propagación con porosidad de la roca.

En procesamiento de datos sísmicos, las velocidades sísmicas de intervalo se correlacionan con las velocidades de propagación $V_{P}$. Esta relación se usa para predecir el tipo de litología a partir de las velocidades sísmicas. La densidad y velocidad de propagación $V_{S}$ pueden conocerse a partir de mediciones en laboratorio o estimarse mediante regresión lineal de información local, usando registros geofísicos de pozos.

Existen diversos modelos de física de roca para diferentes tipos de litología, principalmente para areniscas y carbonatos, mismos que se clasifican en teóricos, empíricos e híbridos (Gutierrez, 2010), cada uno con sus ventajas y desventajas. La mayoría de los modelos empíricos enfatizan el efecto de alguna propiedad, por ejemplo, la variación de porosidad con respecto a la profundidad, temperatura, velocidad o esfuerzo efectivo. Otros modelos enfatizan algún proceso, como el comportamiento mecánico de las rocas que produce valores de porosidad y fracturamiento específicos. Es necesario calibrar los modelos empíricos con información local para que sean efectivos.

Por otra parte, algunos de los modelos teóricos de física de rocas usan teorías de mecánica del medio continuo para estimar las propiedades elásticas de las rocas. Por ejemplo, la teoría de poroelasticidad de Biot-Gassmann generalmente se utiliza para modelar los efectos del fluido. Otros modelos suponen que los 
poros son inclusiones dentro de la roca, por lo que requieren conocer la geometría de los poros para ser efectivos y consideran técnicas matemáticas como la teoría de homogeneización asintótica (Nicolás et al., 2010). Para obtener los beneficios de varios tipos de modelos se crean los llamados modelos híbridos, aunque su mayor limitación es que funcionan mejor en rocas clásticas altamente porosas con fines de interpretación sísmica (Avseth et al., 2010).

Las velocidades se han estudiado principalmente en ambientes sedimentarios clásticos convencionales, esto es, la roca almacén es arenisca y la roca sello es lutita. Con este esquema, se han propuesto diversos modelos físicos de rocas y con ellos se busca correlacionar velocidades con porosidad (Kuster y Toksöz, 1974; Mavko y Mukerji, 1998; Xu y White, 1995). El desarrollo de modelos de física de roca en ambientes sedimentarios profundos, que dan origen a las rocas químicas (por ejemplo, calizas), es extremadamente difícil porque el sistema de poros es mucho más complejo que en rocas clásticas. Esta complejidad de la estructura porosa de los carbonatos produce gran dispersión de los valores de velocidad con respecto a la porosidad, por lo que la ambigüedad es mayor.

Para modelar de manera efectiva un yacimiento de hidrocarburos es indispensable estudiar, además de la litología y la forma de sus poros, las propiedades de los fluidos (fase, densidad, viscosidad, saturación), interacciones roca-fluido (cambios químicos en la roca producidos por los fluidos), anisotropía, entre otros. A continuación presentamos algunos de los factores más importantes que afectan el modelado e interpretación de las velocidades.

\section{Fluido y saturación}

Las rocas saturadas con agua y aceite muestran valores grandes de velocidad $V_{P}$ porque la presencia de fluido incompresible afecta la magnitud del módulo volumétrico. Contrariamente, las rocas saturadas con gas muestran valores pequeños de velocidad por su naturaleza compresible y densidad baja. Con respecto a la velocidad $V_{S}$, las magnitudes son insensibles a la saturación de fluido, ya que éstos no oponen rigidez al esfuerzo de corte. Asimismo, la velocidad $V_{P}$ es insensible para casi cualquier grado de saturación de agua, como se muestra en la figura 5 .

Por otra parte, las propiedades elásticas que afectan la respuesta sísmica de las rocas dependen tanto de las características del fluido como de la estructura sólida o esqueleto. En este sentido, la velocidad de una roca saturada disminuye si existe fracturamiento, ya que éste produce un efecto de incremento de porosidad y la rigidez de la roca es menor (Wang, 2001).

Porosidad y forma de los poros

Empíricamente, la velocidad $V_{P}$ disminuye cuando aumenta la porosidad. Sin embargo, Kuster y Toksöz (1974) demuestran que las propiedades elásticas quedan afectadas más por la forma de los poros que por la porosidad en sí misma. La velocidad de una roca poco porosa, cuyos poros tienen formas aplanadas (como la lutita), muestra un efecto mayor que en una roca muy porosa, de poros esféricos (como la arenisca). La razón es que los poros aplanados son mucho más compresibles que los poros de forma esférica. Esta situación y la propia variación de la forma de los poros en las rocas sedimentarias pueden producir dispersión en las relaciones de velocidad-porosidad. Dado que las rocas son altamente heterogéneas y complejas, especialmente a escala microscópica, las relaciones entre propiedades elásticas y petrofísicas son estrictamente aproximadas y cualitativas en la mayoría de los modelos de física de rocas.

\section{Presión efectiva}

La presión que afecta directamente a las velocidades sísmicas en el yacimiento es la presión efectiva, resultado de la diferencia entre la presión de confinamiento y la presión de poro. Estadísticamente, las velocidades $V_{P}$ y $V_{S}$ incrementan de manera no lineal, al aumentar la presión efectiva. Esto se traduce en una reducción del espacio poroso, ya que las fracturas se cierran al aumentar la presión haciendo la roca más rígida (Gutierrez et al., 2006). Las velocidades muestran un comportamiento asintótico que indica el rango de presión efectiva para el cual las fracturas están totalmente cerradas (figura 5).

Litología y relación $\mathrm{V}_{\mathrm{p}} / \mathrm{V}_{\mathrm{S}}$

Las rocas compuestas por carbonatos son más densas y menos porosas que las rocas formadas por clastos o fragmentos de rocas. Si calculamos la relación entre velocidades para cada litología, observamos que $V_{P} / V_{S}$ es mayor para las calizas que para areniscas. Mientras que para lutitas, que muestran valores en un rango amplio de velocidades, siempre muestran valores más grandes que las areniscas en cuanto a la relación de velocidades $V_{P} / V_{S}$ (Wang, 2001).

Tradicionalmente, la relación $V_{P} / V_{S}$ se utiliza como indicador de la presencia de hidrocarburos a través del 
análisis de la variación de amplitud con la distancia ( $A V O$ por sus siglas en inglés). Su aplicación responde a la incapacidad de las ondas S para propagarse por medios fluidos, generando así cambios notorios en la relación $V_{P} / V_{S}$ cuando la roca está saturada. También es práctica común utilizar relaciones lineales entre $V_{P}$ y $V_{S}$ para simplificar el problema cuando el acceso a mediciones experimentales es limitado o inexistente; sin embargo, con ello se elimina la variación intrínseca de las velocidades producida por cambios de facies. Actualmente, las nuevas prácticas incorporan caracterización de rocas en laboratorio en sus metodologías para hacerlas más confiables.

Resumiendo, modelar velocidades permite caracterizar tipos de roca e investigar la respuesta sísmica para diversos escenarios geológicos y, con ello, crear un catálogo de respuestas sísmicas asociadas a zonas con potencial exploratorio y a escenarios sin atractivo económico.

De esta manera, la interpretación de las velocidades sísmicas permite caracterizar los yacimientos en términos de propiedades de las rocas y para extrapolar (predecir) propiedades petrofísicas en regiones alejadas de los pozos. Este último objetivo involucra integrar diferentes tipos de información y estimar rangos de incertidumbre para crear modelos de yacimiento más confiables.

\section{Integración de datos para predicción de propie- dades de la roca}

Idealmente, los modelos físicos de rocas podrían utilizarse para determinar propiedades y condiciones del yacimiento (porosidad, litología, presión, temperatura), directamente de los datos sísmicos. Sin embargo, es necesario evaluar primero la aplicabilidad de dichos modelos físicos fuera del rango de resolución en que éstos fueron diseñados. Esto es, las ondas elásticas sísmicas observan porciones grandes del subsuelo, cuyas dimensiones son, al menos, dos órdenes de magnitud mayores a las observadas con registros geofísicos de pozos (figura 1). Así, las imágenes sísmicas están asociadas cualitativamente con las propiedades efectivas del subsuelo, por lo que los detalles de menor escala obtenidos con registros de pozos son imposibles de recuperar mediante datos sísmicos.

Para evaluar la aplicabilidad de las relaciones de transformación obtenidas del análisis de física de rocas es necesario escalar o remuestrear (del término upscaling) las curvas de los registros de pozos mediante el método de promedios de Backus (1962). Esta técnica consiste en promediar los módulos elásticos (no las velocidades sónicas) y densidad calculados con registros de pozos, con el fin de estimar las propiedades elásticas a escala sísmica (figura 6).

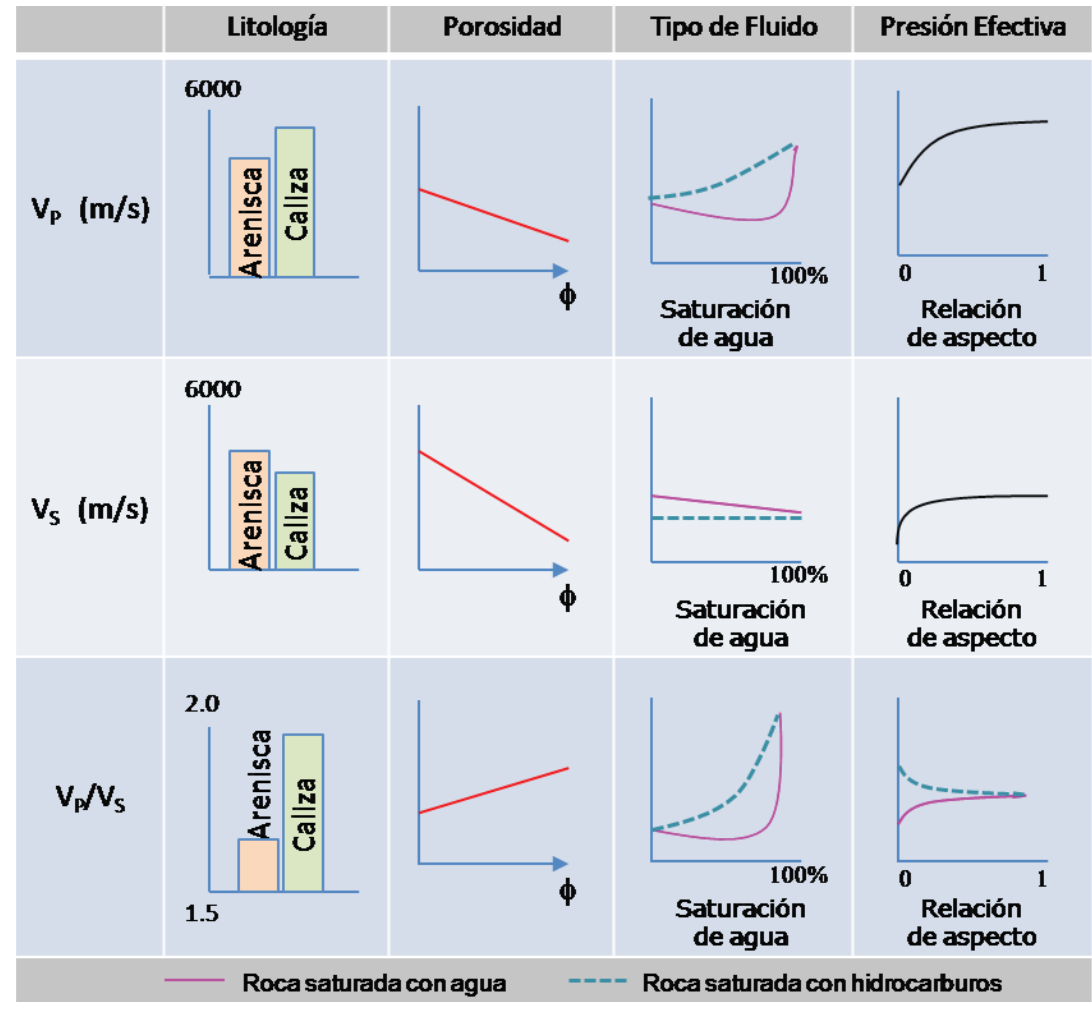

Figura 5. Variación de las velocidades $V_{p}, V_{S}$ y la relación $V_{p} / V_{S}$ en función de la litología, porosidad, tipo de fluido y presión efectiva 


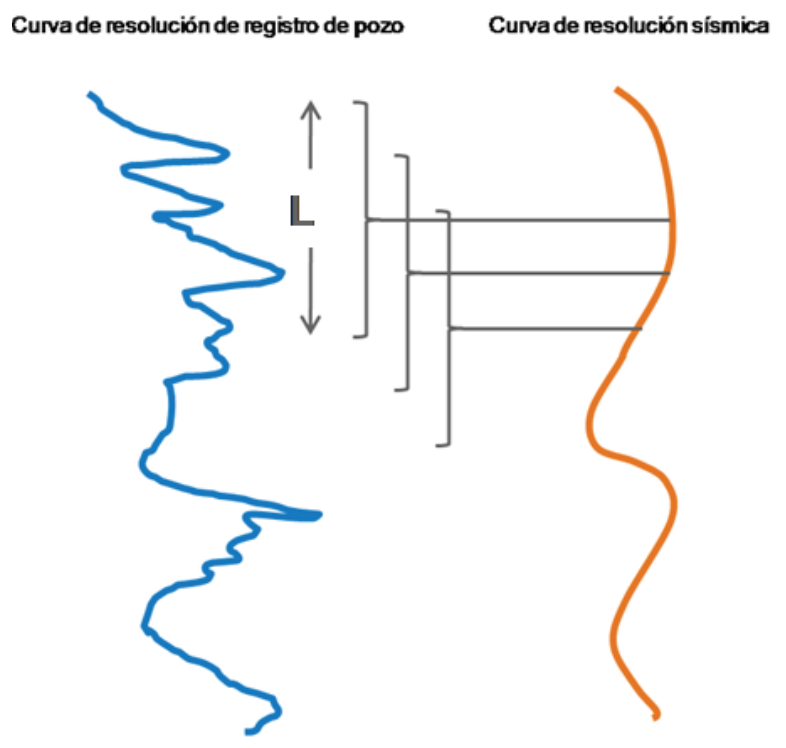

Figura 6. La técnica de Backus (1962) escala propiedades elásticas de la roca observadas en pozo para obtener valores de velocidad $V_{p}$ y $V_{S}$ en el rango de escala sísmica, mediante una ventana de escalamiento de longitud $L$ (modificado de Lindsay y Van, 2001)

Generalmente, las propiedades volumétricas de la roca, por ejemplo, densidad, porosidad, se escalan mediante el promedio aritmético, mientras que para los módulos elásticos, como los parámetros de Lamé $\mu$ y $\lambda$, se utiliza el promedio armónico como se indica en las ecuaciones 12 y 13 ,

$\langle P\rangle={ }^{N} \sqrt{\prod_{i=1}^{N} P_{i}}$

$\langle M\rangle=\left(\sum_{i=1}^{N} \frac{N}{M_{i}}\right)$

donde

$\langle P\rangle$ es el promedio de cualquier propiedad volumétrica,

$\langle M\rangle$ el promedio de cualquiera de los parámetros de Lamé,

$N$ es el número total de muestras,

$i \quad$ es el número de muestra.

Estas expresiones se generalizan y pueden variar dependiendo de la aplicación. La teoría de Backus se considera una técnica para suavizar velocidades sónicas, no obstante, es un método para determinar parámetros de anisotropía sísmica (Liner y Fei, 2007).
Remuestrear las curvas de propiedades geofísicas requiere de una ventana de escalamiento definida en profundidad, porque los registros de pozo están registrados en profundidad. Generalmente, la longitud de la ventana de escalamiento $L$ se define con base en las características de la ondícula sísmica extraída de los datos sísmicos.

Las características de esta ondícula sísmica (longitud y frecuencia dominante) cambian dinámicamente, por lo que la ventana de escalamiento debe cambiar dinámicamente también, con el fin de preservar las interfaces graduales de velocidad (Lindsay y Van, 2001).

Para convertir la longitud de la ventana de tiempo a profundidad se usa la velocidad sísmica $V_{P}$ promedio a lo largo de la ventana, como se muestra en la figura 6 . Una vez escalados los registros de módulos elásticos, se calculan los valores de velocidad correspondientes mediante las ecuaciones 2 y 3 , de manera inversa.

En síntesis, las relaciones de física de rocas escaladas no pueden ser generalizadas porque las propiedades escaladas pueden estar fuertemente influidas por el tamaño y forma del yacimiento y la litología que lo circunda. Validar las relaciones de física de rocas a escala sísmica es más confiable a medida que el espesor del yacimiento es mayor y la variación de propiedades de la roca, en profundidad, alrededor del yacimiento sea gradual.

\section{Avances tecnológicos y aplicaciones}

Estudio de lutitas y anisotropía

Recientemente los estudios petrofísicos han permitido reconocer la gran complejidad de las lutitas y han detallado información nueva y relevante para el análisis de datos sísmicos, sobre todo de yacimientos no convencionales (Smith et al., 2009; Prasad et al., 2009). Para interpretar los contrastes de amplitud sísmica en términos de propiedades de las rocas es necesario analizar el comportamiento de las lutitas por medio de física de rocas.

Esto requiere, necesariamente, modelos más complejos que incluyan detalles sobre microfracturas, microporosidad, minerales diagenéticos $\mathrm{y}$, particularmente, anisotropía. Con ello, el desarrollo de metodologías experimentales para medir propiedades de los minerales arcillosos ha incrementado a medida que se obtiene un mayor número de muestra de roca durante la perforación.

Este rubro tiene un gran potencial de aplicación, por ejemplo, en la explotación de yacimientos de gas en lutitas. 
Predicción de presión de poro y estabilidad del pozo

Una de las aplicaciones del análisis de física de rocas es la predicción de presión de poro y esfuerzo efectivo del yacimiento para diseño de perforación de un pozo. Algunos métodos para estimar estas propiedades realizan inversión de velocidades sísmicas de intervalo (Bruce y Bowers, 2002; Mendoza et al., 2009), otros métodos realizan modelado geológico de cuencas para cuantificar la presión de confinamiento o bien a partir de relaciones entre porosidad, velocidad sísmica y profundidad (Gutierrez et al., 2006).

\section{Cuantificación de incertidumbre por métodos geoestadísticos}

Tradicionalmente, la distribución de litología o porosidad se estima a partir de los datos sísmicos en dos etapas:

1) Inversión de datos sísmicos antes de apilar para estimar propiedades elásticas del subsuelo y

2) Transformación de los volúmenes de atributos sísmicos en indicadores de litología y porosidad mediante regresión lineal o técnicas de reconocimiento guiadas por los datos sísmicos (Avseth et al., 2007).

El problema de escalamiento, debido al contenido de frecuencias limitado de los datos sísmicos y a la conversión de datos de profundidad a tiempo, puede generar inconsistencias con el modelo de velocidad sísmico (Doyen, 2009).

Actualmente se usan modelos petroelásticos para hacer modelado de velocidades $V_{P}$ y $V_{S}$ a partir de propiedades de la roca. Estos modelos petroelásticos reúnen relaciones físicas y empíricas, cuyos parámetros están asociados a las propiedades elásticas de los componentes minerales, propiedades del fluido, textura de la roca, profundidad del yacimiento, etcétera.

Una manera de cuantificar el grado de incertidumbre de las estimaciones de propiedades del yacimiento a partir de datos sísmicos es por medio de la geoestadística. El análisis estadístico de física de rocas fue introducido por Mavko y Mukerji (1998) y Avseth et al. (2007), entre otros, para cuantificar la caracterización sísmica de yacimientos con fines de producción. En este tipo de análisis se combinan relaciones deterministas y geoestadísticas, donde las primeras se usan para establecer las reglas de transformación entre las propiedades del yacimiento y las propiedades sísmicas, mientras que las segundas se usan para propagar la incertidumbre asociada a la transformación de una propiedad a otra, a la vez que describe la variabilidad espacial de las propiedades.

Generalmente, en un estudio exploratorio se cuenta con muy pocos pozos para representar apropiadamente las diferentes litologías o fluidos presentes en el subsuelo. Una solución a la falta de datos es generar información estadística, con algoritmos de simulación numérica, a partir de modelos de física de rocas para calcular propiedades elásticas y atributos sísmicos, usando información petrofísica de los pozos (Doyen, 2009). Con la nueva información se puede calcular un modelo promedio y la deviación estándar y así obtener intervalos de incertidumbre.

\section{Modelos digitales de física de rocas}

La información más confiable es la que se mide directamente en la roca (en condiciones de yacimiento). Realizar estas mediciones puede ser problemático si no se realiza sobre la misma muestra de roca o si las muestras son escasas y las mediciones consumen mucho tiempo. Idealmente, se requieren mediciones para cientos o miles de muestras, pero en la práctica difícilmente disponemos de ello. Una manera de obtener abundancia de datos es el análisis digital de física de rocas (Knackstedt et al., 2009).

Con este método se obtienen imágenes digitales en dos y tres dimensiones de la estructura interna de muestras de roca, que permiten calcular propiedades elásticas y simular procesos a escala micrométrica rápidamente. Los modelos digitales se generan a partir de láminas delgadas de la muestra de roca o por escaneo digital. La geometría de la estructura interna se modela entonces con algoritmos, por ejemplo, de diferencias finitas. De esta manera, se puede caracterizar un medio poroso que permite observar los fenómenos físicos, e incluso químicos, que se presentan en la roca y los efectos simultáneos que ocurren durante el modelado de propiedades elásticas.

Por último, la aplicación del análisis cuantitativo de física de rocas no se limita a la exploración de hidrocarburos, también tiene extensa aplicación en problemas de producción y desarrollo de pozos, recuperación secundaria, simulación de yacimientos y sismología de lapso.

\section{Conclusiones}

Se revisaron brevemente los fundamentos del análisis de física de rocas para fines de exploración de hidrocarburos y algunas de las más importantes aplicaciones usando datos sísmicos, pues esta práctica multidiscipli- 
naria adquiere relevancia a medida que los yacimientos son más complejos. La aplicación del conocimiento detallado de las propiedades elásticas de las rocas asociadas con los datos sísmicos permite no sólo mejorar las actividades de exploración sino monitorear el desarrollo de campos petroleros a través de información sísmica en diferentes etapas de la explotación.

Los avances se han reflejado en esfuerzos de modelado numérico de roca y mediciones experimentales en laboratorio, utilizando varias técnicas independientes y la integración de información para interpretación tomando en cuenta los diferentes rangos de frecuencia. Se debe tomar en cuenta el efecto sobre las propiedades de las rocas de diferentes factores, como el tipo de fluido, porosidad, nivel de esfuerzo efectivo, grado de saturación y tipo de litología. Los modelos físicos de rocas se pueden utilizar como modelos predictivos de propiedades petrofísicas y aplicarse directamente en los datos sísmicos, siempre y cuando se consideren las diferencias de escala en frecuencia de las diferentes informaciones. Asimismo, se enfatiza el impacto de incluir mediciones experimentales del comportamiento geomecánico como un estándar en los flujos de trabajo convencionales, así como alternativas computacionales y estadísticas para cuantificación de geometrías y propiedades mecánicas, respectivamente. Todo lo anterior define el sentido litológico cuantitativo de la información sísmica que optimiza las actividades de exploración de hidrocarburos.

En el contexto experimental se presentaron dos técnicas de laboratorio independientes para caracterizar núcleos de roca: la técnica de resonancia en el dominio de la frecuencia y las mediciones ultrasónicas en el dominio de tiempo. Ambas proporcionan información sobre la rigidez de las rocas en diferentes rangos de frecuencia y deformación, por lo que se contribuye a la redundancia experimental y a la posible extrapolación de información. Adicionalmente, las pruebas de resonancia permiten evaluar el amortiguamiento del material, por lo tanto, estimar el factor de calidad $Q$.

Finalmente se presentaron diferentes aplicaciones de la física de rocas al estudio de la anisotropía, predicción de presión de poro, así como la aplicación de métodos estadísticos y de confiabilidad para caracterizar probabilísticamente las propiedades de las rocas. La relevancia de incorporar el estudio de condiciones de anisotropía sobre las propiedades de las rocas es crucial para la correcta exploración de sitios con geología compleja, como el caso de yacimientos no convencionales en lutitas. El estudio de presión de poro definitivamente se beneficiaría si el modelo de velocidades aumenta su nivel de resolución con un sentido completamente litológico.

\section{Agradecimientos}

Los autores agradecen el apoyo y financiamiento a la Gerencia de Geofísica (GGf) de PEMEX, Exploración y Producción (PEP) para la elaboración de cursos y material didáctico. Particularmente a Marco Vázquez García, Otila Mayes Mellado y Gerardo Clemente por sugerir el tema y sus comentarios. Agradecemos también al personal técnico de los diferentes Activos de PEP por su participación durante los talleres tecnológicos realizados en 2009 y 2010.

\section{Referencias}

Avila-Carrera R., Spurlin J.H., Valle-Molina C. Simulating Elastic Wave Propagation in Boreholes: Fundamentals of Seismic Response and Quantitative Interpretation of Well-Log Data. Geofísica Internacional, volumen 50 (número 1), 2011: 57-76.

Avseth P., Mukerji T., Mavko G., Dvorkin J. Rock-Physics Diagnostics of Depositional Texture, Diagenetic Alterations and Reservoir Heterogeneity in High-Porosity Siliciclastic Sediments and Rocks - A Review of Selected Models and Suggested Work Flows. Geophysics, volumen 76 (número 6), 2010: 75A31-75A47.

Avseth P., Mukerji T., Mavko G. Quantitative Seismic InterpretationApplying Rock Physics Tools to Reduce Interpretation Risk, Cambridge University Press, 2007.

Backus G.E. Long-Wave Elastic Anisotropy Produced by Horizontal Layering. Journal of Geophysical Research, volumen 67 (número 11), 1962: 4427-4440.

Bruce B., Bowers G. Pore Pressure Terminology. The Leading Edge, volumen 26, febrero , 2002: 170-173.

Doyen P.M. Seismic Reservoir Characterization: An Earth Modeling Perspective, EAGE (European Association of Geoscientists \& Engineers) Publications, The Netherlands, 2009.

Dvorkin J., Nur A. Scale of Experiment and Rock Physics Trends. The Leading Edge, volumen 28, 2009: 110-115.

Gutierrez M.A. Rock Physics Workflows for Exploration in Frontier Basins. Expanded Abstracts, SEG Denver, EU, 2010.

Gutierrez M.A, Braunsdorf N.R., Couzens B.A. Calibration and Ranking of Pore Pressure Prediction Models. The Leading Edge, volumen 25 (número 12), 2006: 1496-1500.

Harris J.M., Langan R.T. Crosswell Seismic Fills the Gap. AAPG Explorer, volumen 18 (número 1), 1997: 10-12.

King M.S. Recent Developments in Seismic Rock Physics. International Journal of Rock Mechanics and Mining Sciences, volumen 46, 2009: 1341-1348.

Kemper M. Rock Physics Driven Inversion: the Importance of Workflow. First Break, volumen 28, 2010: 69-81.

Knackstedt M.A., Latham S., Madadi M., Sheppard A., Varslot T., Arns C. Digital Rock Physics: 3D Imaging of Core Material and Correlations to Acoustic and Flow Properties. The Leading Edge, volumen 28, 2009: 28-33. 
Kuster G.T., Toksöz M.N. Velocity and Attenuation of Seismic Waves in Two-Phase Media, Part I, Theoretical Formulations and Part II, Experimental Results. Geophysics, volumen 39, 1974: 587-618.

Liner C., Fei T. The Backus Number. The Leading Edge, volumen 26, 2007: 420-426.

Lindsay R., Koughnet V. Sequential Backus Averaging-Upscaling well Logs to Seismic Wavelengths. The Leading Edge, volumen 20, 2001:188-191.

Mavko G., Mukerji T. A Rock Physics Strategy for Quantifying Uncertainty in Common Hydrocarbon Indicators. Geophysics volumen 63, 1998: 1997-2008.

Mendoza-Amuchástegui J., Vázquez-Jiménez G., Espinosa-Ortega M., Valle-Molina C., Alvarado-Hernández E., García-Herrera M., Nicolas-López R. Pore-Pressure Prediction and Wellbore Stability in the Deep Mexican Gulf of Mexico. The Leading Edge, volumen 28, 2009: 702-706.

Nicolás-Lopez R., Valdiviezo-Mijangos O., Valle-Molina C. New Approach to Calculate the Mud Density for Wellbore Stability Using the Asymptotic Homogenization Theory. Petroleum Science and Technology, volumen 30, 2012: 1239-1249.

Prasad M., Pal-Bathija A., Johnston M., Rydzy M., Batzle M. Rock Physics of the Unconventional. The Leading Edge, volumen 28, 2009: 34-38.

Smith T.M., Sayers C.M. y Sondergeld C.H. Rock Properties in Low-Porosity/Low-Permeability Sandstones. The Leading Edge, volumen 28, 2009: 48-59.

Stokoe K.H., Hwang S.K., Lee J.N., Andrus R.D. Effects of Various Parameters on the Stiffness and Damping of Soils at Small to Medium Strains, in: Proceedings, International Symposium on Pre-Failure Deformation Characteristics of Geomaterials, volumen I, Japanese Society of Soil Mechanics and Foundation Engineering, Sapporo, Japón, 1994a, pp. 785-816.

Stokoe K.H., Hwang S.K., Roesset J.M. Laboratory Measurements of Small-Strain Material Damping of Soil Using a Free-Free
Resonant Column. $2^{\text {nd }}$ Int. Conf. on Earthquake Resistant and Construction and Design, Germany, 1994b, pp.195-202.

Wang Z. Fundamentals of Seismic Rock Physics. Geophysics, volumen 66, 2001: 398-412.

Xin-Gang C., De-Hua H. Lithology and Fluid Differentiation Using a Rock Physics Template. The Leading Edge, volumen 28, 2009: 60-65.

Xu S., White R.E. A New Velocity Model for Clay-Sand Mixtures. Geophysical Prospecting, volumen 43, 1995: 91-118.

\section{Bibliografía}

Goodman R.E. Introduction to Rock Mechanics, John Wiley \& Sons, 1989.

Jaeger J.C., Cook N.G.W. Fundamentals of Rock Mechanics, Chapman \& Hall, 1979.

Guéguen Y., Palciauskas V. Introduction to the Physics of Rocks, Princeton University Press, 1994.

Tiab D., Donaldson E.C. Petrophysics: Theory and Practice of Measuring Reservoir Rock and Fluid Transport Properties, Gulf Publishing Company, 1996.

\section{Este artículo se cita:}

\section{Citación Chicago}

Vargas-Meleza, Liliana, Celestino Valle-Molina. Avances y aplicaciones en física de rocas para exploración de hidrocarburos. Ingeniería Investigación y Tecnología XIII, 04 (2012): 439-451.

\section{Citación ISO 690}

Vargas-Meleza L., Valle-Molina C. Avances y aplicaciones en física de rocas para exploración de hidrocarburos. Ingeniería Investigación y Tecnología, volumen XIII (número 4), octubre-diciembre 2012: 439-451.

\section{Semblanza de los autores}

Liliana Vargas-Meleza. Obtuvo el grado de ingeniera en geofísica por la Facultad de Ingeniería de la UNAM en 2003 y el grado de maestra en geofísica aplicada, tanto por la Universidad de Tecnología de Delft (TUDelft), Países Bajos, el Instituto Federal Suizo de Tecnología en Zurich (ETHZ), como por la Universidad de Aachen (RWTH), Alemania, en 2009, con especialidad en sismología de exploración. Especialista en el Departamento de Prospección Geofísica del Instituto Mexicano del Petróleo desde 2003. Sus áreas de interés son inversión petrofísica y representación sísmica de cuerpos salinos. Desde 2011, realiza estudios doctorales en la Universidad de Aberdeen, Reino Unido.

Celestino Valle-Molina. Ingeniero civil y maestro en ingeniería en mecánica de suelos por la Universidad Nacional Autónoma de México (UNAM) en 1995 y 1997, respectivamente. Es doctor en ingeniería (Ph.D) con especialidad en geotecnia por la Universidad de Texas en Austin, EU en 2006. Es investigador del Instituto Mexicano del Petróleo (IMP) desde 2007. Sus áreas de interés profesional abarcan dinámica de suelos y rocas, ingeniería geotécnica fuera de la costa, geomecánica aplicada a exploración petrolera y confiabilidad aplicada a ingeniería geotécnica. Es profesor de asignatura en la Escuela Superior de Ingeniería y Arquitectura (ESIA) del Instituto Politécnico Nacional (IPN) en su Sección de Estudios de Posgrado e Investigación desde 2006. 